\section{Incomplete Dissociation in Solutions of Strong Electrolytes}

Kasimir Fajans

Depariment of Chemistry, University of Michigan, Ann Arbor, Michigan February 14, 1941

CINCE 1927 the view has been held ${ }^{1}$ that the change $\mathcal{O}$ of the apparent molar refraction of strong electrolytes with concentration is due to the formation of combinations of oppositely charged ions coming in direct contact with each other without water molecules between them, i.e., due to the formation of undissociated particles. ${ }^{2}$ The investigation of the apparent molar dispersion ${ }^{3}$ gave an additional strong support for this view as does, according to Neugebauer, ${ }^{4}$ his result that the refractometric effects are due to the interpenetration of the electronic systems.

The preceding letter leads to the conclusion that the formation of undissociated particles should be accompanied by a change of volume of the ions themselves.

As has been known for a long time, in the infinitely dilute solution the attraction of the water by the ions causes a contraction of the surrounding water (electrostriction) thus diminishing the apparent volume $(\Phi)$ of the electrolyte.

When the solution becomes concentrated two effects are involved which should cause an increase of $\Phi$ for a completely dissociated electrolyte consisting of rigid ions: The electrostriction of the water removed is eliminated; the electric field of the ions is partly screened and its contracting action on the remaining water diminished due to the formation of Debye's ionic atmosphere.

The theory of the influence of the Debye effect ${ }^{5}$ gives for small concentrations a limiting linear relation between $\Phi$ and $\sqrt{ } c$, the slope of which is $1.9 \mathrm{cc} /($ mole/liter) for mono-monovalent electrolytes at $25^{\circ} \mathrm{C}$.

It has been found ${ }^{6}$ that this theoretical slope holds for $\mathrm{NaCl}$ up to $0.4 \mathrm{~N}$, for higher concentrations the effect shows individual behavior for different electrolytes although the relation becomes again linear. ${ }^{7}$ Figure 1 gives a small selection of the available data. ${ }^{8}$

One can expect that if the individual behavior of different electrolytes were due exclusively to interionic forces between rigid ions those electrolytes which have equal osmotic or activity coefficients would come near each other also in respect to their slopes for the $\Phi v s . \sqrt{ } c$ lines. This is not at all the case; e.g., among the electrolytes given in Fig. $1, \mathrm{KF}$ and $\mathrm{NaBr}$ have nearly equal osmotic coefficients but the slope of $\mathrm{KF}$ (3.35) in Fig. 1 is very much larger than that of $\mathrm{NaBr}(1.80)$. The comparison of a large number of cases with similar coefficients shows that the factor which is responsible for this discrepancy is the influence of the mutual polarization of the ions on their volumes.

A comparison of Fig. 1 with Fig. 1b (see page 281) shows at once that those salts for which the apparent volume increases strongly when their ions are brought from the infinitely dilute solution into the direct contact in the crystal have the largest slopes in Fig. 1. On the other hand LiI which is at the bottom of Fig. $1 \mathrm{~b}$ on page 281 has the smallest initial slope among the salts. The simplest conclusion is that the change of volume

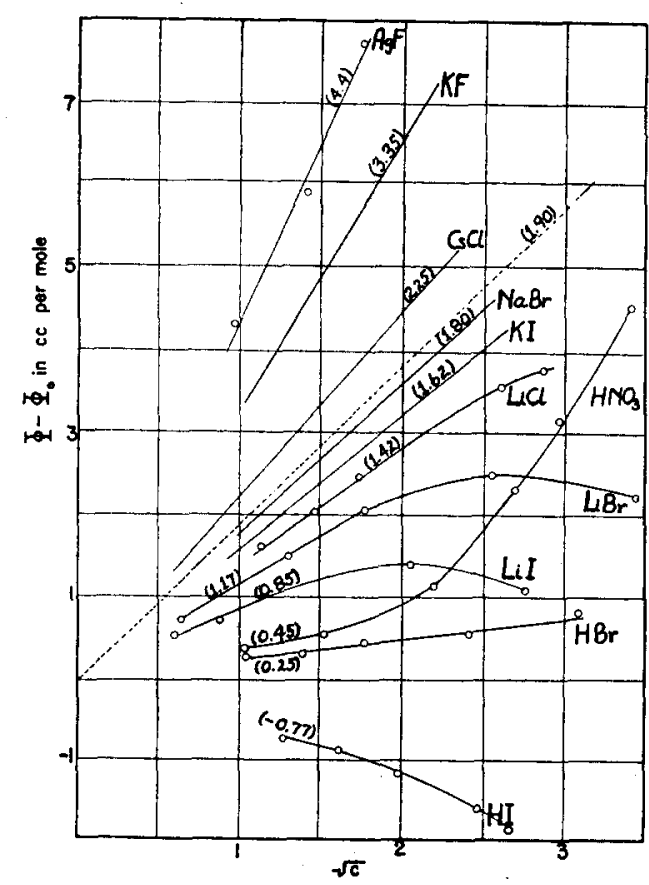

FIG. 1. Difference between the apparent molar volume at the molarity $c$ and at infinite dilution as a function of $\checkmark c$. The numbers on the $c$ and at infinite dilution as a function of
curves refer to the slope in $\mathrm{cc} /$ (mole, liter).

in concentrating the solution is due not only to the screening of the field of the ions but also to an individual effect caused by their interpenetration. The strongest evidence, however, which can be derived from Fig. 1 for the conclusion that concentrated solutions of strong electrolytes contain appreciable amounts of undissociated particles is given by the behavior of $\mathrm{HI}$, for which a considerable negative slope was found. ${ }^{9}$

It was mentioned in the preceding letter that the introduction of the proton into the iodide ion diminishes its refraction and consequently its volume by about one-third of the initial value. A mere association of $\mathrm{OH}_{3}{ }^{+}$with $\mathrm{I}^{-}$ must also cause a very considerable effect in the same direction. Thus the negative slope of the HI curve must be explained by the predominance of one or both of these effects over the effect of the diminution of the electrostriction. The small cation $\mathrm{Li}^{+}$is next to the proton in the deforming power and we see in Fig. 1 that the curves for $\mathrm{LiI}$ and $\mathrm{LiBr}$, after an initial rise, go through a maximum and fall at high concentrations.

I thank Mr. Norman Bauer very much for valuable discussion.

1 K. Fajans, Trans. Faraday Soc. 23, 357 (1927). List of later papers cf. reference 1 of preceding letter. "Polarization of Ions and Lattice Distances."

2 See however, A. I. Brodsky, Zh. M. Shershever and N. S. Filippova, Chem. Abstracts 33, 9074 (1939).

3 Cf. A. Kruis, reference 1, of preceding letter on "Polarization of Ions and Lattice Distances."

4See reference 9 of preceding letter entitled "Molar Volume, Refraction and Interionic Forces."

$5 \mathrm{O}$. Redlich and P. Rosenfeld, Zeits. f. Elektrochemie 37, 705 (1931). A. Kruis, Zeits. f. physik. Chemie B34, 1 (1936)

7 O. Masson, Phil. Mag. [7] 8, 218 (1929). W. Geffcken, Zeits. f. physik. Chemie A155, 1 (1931).

8 The values for alkali halides are taken from the paper of W. Geffcken (reference 7). For calculation of the other values, as well as of many data on which the conclusions of this and the above letter are based, $\mathrm{I}$ am very much indebted to Mr. Oliver Johnson.

9 R. Lühdemann, Zeits. f. physik. Chemie B29, 133 (1935). 